\title{
LHeC: The Large Hadron-Electron Collider
}

\author{
John Dainton * \\ The Cockcroft Institute of Accelerator Science and Technology, \\ Daresbury Science and Innovation Campus, Warrington WA4 4AD, UK, \\ and Department of Physics, University of Liverpool, UK
}

\begin{abstract}
Progress towards an upgrade, the $\mathrm{LHeC}$, of the $\mathrm{LHC}$ at CERN is presented in which electron(positron)-proton (ep) physics at the TeV energy scale is realized by bringing a $70 \mathrm{GeV}$ electron(positron) beam into collision with a $7 \mathrm{TeV}$ hadron beam in the LHC tunnel. An ep luminosity of $10^{33} \mathrm{~cm}^{-2} \mathrm{~s}^{-1}$ seems feasible. An update on the physics horizon is outlined. Aspects of a first evaluation of the possibility of an $e$-storage ring are presented in which the aim of simultaneous $p p$ (ion-ion $A A$ ) and $e p(e A)$ data-taking is achieved. A putative timeline for the realization of an $\mathrm{LHeC}$ is mentioned.
\end{abstract}

\section{Why a Universe with both Leptons and Quarks?}

In 1967 our understanding of the fundamental nature and structure of matter posed a conundrum: why did matter at the femtometric scale distinguish itself as a tidy array of only two generations of "point-like", Dirac, fermions - the leptons $e$ and $\mu$ - and a complicated spectrum of hadrons, characterized by those which could decay through the strong interaction to nucleons (non-strange hadrons) and those which couldn't (strange hadrons)?

By 1969, we had, through definitive measurements at the Stanford Linear Accelerator Collider (SLAC), clear evidence that a sub-femtometric view simplifies the hadronic spectrum to that of also of point-like, Dirac, fermions - quarks - as postulated by Gellmann and Zweig [1], and as interpreted by Bjørken and Feynman [2]. Deep-inelastic, lepton-nucleon, scattering had brought particle physics to a turning point. There followed decades of staggering progress which now culminates in today's Standard Model, and its huge impact on Cosmology and our understanding of our place in the Universe.

The definitive measurements at SLAC were of course those of the Nobel Prize-winning experiment at end-station A. There, the first measurements of deep-inelastic, lepton-nucleon, scattering were made, and the first cross sections (structure functions) obtained from the data [3]. The SLAC experiment was revolutionary not because of its centre-of-mass (CM) energy, for there were fixed target, hadron-beam, experiments at CERN, and later at Fermilab, with similar or significantly greater energy ${ }^{\mathrm{a}}$. The SLAC experiment's impact was due to the combination of enough interaction energy and enough luminosity to populate the final state phase space where there is sensitivity to short distance structure in the nucleon: the revolutionary discoveries made were with a probe of spatial resolution of about ${ }^{1} / 10 \mathrm{fm}$, that is of about $1 / 10$ th of the size of the target proton.

\footnotetext{
*Email contact with the emerging organization concerned with progressing the $\mathrm{LHeC}$ initiative is best made to mklein@hep.ph.liv.ac.uk or J.B.Dainton@cockcroft.ac.uk, or through the web page http://epweb2.ph.bham.ac.uk/user/newman/LHeC/LHeC.html

a It is worth recalling that at the time of the SLAC end-station-A discoveries in 1969 with a $20 \mathrm{GeV} / \mathrm{c}$ electron beam $-6 \mathrm{GeV}$ centre-of-momentum $(\mathrm{CM})$, the horizon for the energy frontier was $p p$ physics at the CERN ISR with a CM energy of $63 \mathrm{GeV}$. Furthermore, in $p p$ collisions hadronic jets, with a $p_{T}$ dependence attributable to parton-parton scattering, were only unambiguously confirmed more than a decade later in the early 1980s at the CERN SppS collider with a CM energy of $630 \mathrm{GeV}$, a factor $\sim 30$ larger than the original SLAC ep measurements.
} 
Today further deep-inelastic, lepton-hadron experiments, culminating with those at HERA, have enabled us to quantify the chromodynamic picture of hadronic matter down to $1 \mathrm{am}$ distance scale (about ${ }^{1} / 1000$ of the size of stable, colour singlet, hadrons - fig.1). Furthermore, we are now able to probe the kinematic region of low Bjørken- $x$ where proton structure is almost exclusively attributable to chromodynamic field energy. Yet despite this progress, little further understanding has emerged as to why there are three generations of point-like, Dirac, leptons, which remain distinct from three generations of point-like, Dirac, quarks. We inherit the original conundrum of the 1960s, but now in a form which has moved on to address hadronic matter at the attometric scale in terms of quarks and chromodynamics, and their relation to

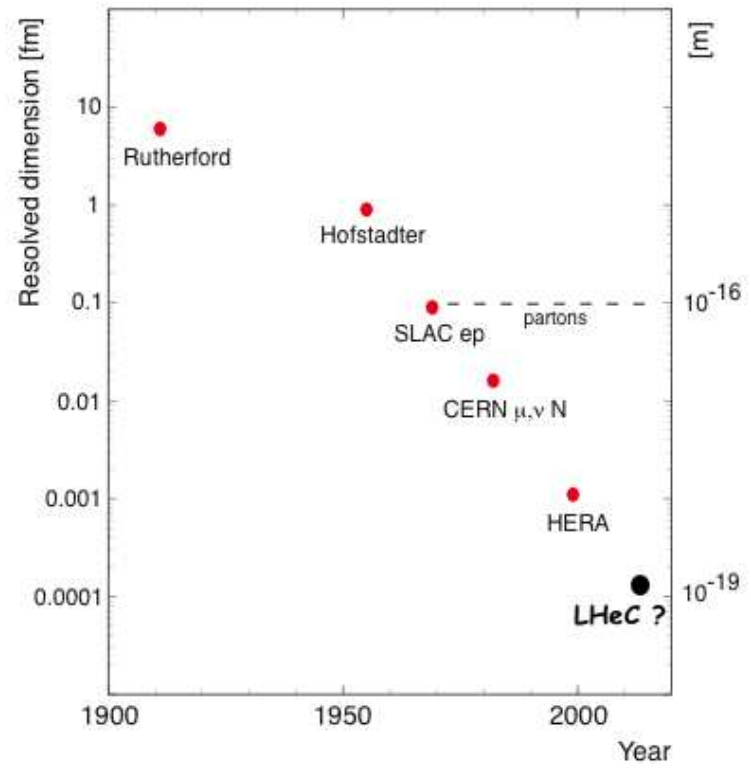

Figure 1: Resolution of lepton probe in "milestone", deep-inelastic, experiments in the $20^{\text {th }}$ century, including that foreseen at the $\mathrm{LHe}$ in the $21^{\text {st }}$ century.

leptons. The question remains one of, if not the, fundamental challenges of all of physics [4].

Our (for I am not alone [5]) purpose here is to remind you that we can now contemplate a new turning point in lepton-hadron physics of significance similar to that following the pioneering SLAC experiment in 1969. Without huge new investment at the soon-to-becompleted LHC, it seems feasible to contemplate an upgrade, the $\mathrm{LHeC}$, for $\mathrm{TeV}$, deepinelastic, $e$-hadron ( $e p$ and $e A$ ) physics, while at the same time retaining on-going, $14 \mathrm{TeV}$, $p p$ and $A A$ experiments.

All that is missing is a $70 \mathrm{GeV}$ (or more) electron or positron beam optimized for collisions of $e$-bunches with LHC $p$-bunches. Such an electron/positron beam at CERN, whether stored in the LHC tunnel or from a high duty cycle (CW), single pass, linac, is a feasible proposition.

We here concern ourselves primarily with an overview of the physics which must be addressed at an $\mathrm{LHe}$ C when electron and positron-quark $(e q)$ collisions at $\mathrm{TeV}$ energy are achieved. We motivate a proposal which takes advantage of the energy and luminosity possible when the LHC hadron beams, $p$ and $A$, are brought into collision with a stored electron or positron beam of realistic intensity. We demonstrate the feasibility of such a configuration as an upgrade of the LHC in which both $e p$ and $p p$, or alternatively both $e A$ and $A A$, physics can be pursued simultaneously. The unprecedented combination of $\mathrm{CM}$ energy and luminosity which is then achieved makes possible measurements of deepinelastic, lepton-hadron, scattering with a spatial resolution of about $1 / 10 \mathrm{am}$, that is of about $1 / 10,000$ th of the dimension of a hadron, amounting to more than a factor 10 better than hitherto (HERA) (fig. 1). 


\section{Lepton-Hadron TeV Physics at the $\mathrm{LH} e \mathrm{C}$}

There have been a number of considerations of how best to achieve TeV electron-proton and electron-ion ${ }^{\mathrm{b}}$ collisions, stemming initially from the, perhaps fanciful, idea of keeping LEP assembled and ready while installing and then operating the LHC. They stand or fall on the basis by which the pioneering SLAC experiments at end station A changed the face of physics, namely by having both adequate energy and adequate luminosity for at least an order of magnitude increase of spatial resolution (fig.1).

It is instructive to take a look at lepton-hadron experiments, both completed and contemplated, in terms of luminosity (fig. 2). Taking advantage of $\mathrm{R} \& \mathrm{D}$ for $\mathrm{TeV} e^{+} e^{-}$physics, whenever the collision of a $250 \mathrm{GeV}$, International Linear Collider (ILC)-like, beam with a co-located $\mathrm{TeV}$ hadron beam, for example at HERA, is considered, luminosity has been the limiting issue (THERA, fig. 2) [6]. This is also the case when the collision of a futuristic, CLIC-technology based (few hundred $\mathrm{GeV}$,or even $\mathrm{TeV}, 30 \mathrm{GHz}$ ), electron beam with a "super-bunched" LHC proton beam is considered (QCD Explorer, fig. 2) [7].Taking the LHC $p$ and $A$ beams as for $p p$ and $A A$ physics, an electron storage ring of energy 70 to $100 \mathrm{GeV}$ in the LHC tunnel, whose design makes possible efficient bunch-crossing with the LHC beams and whose power consumption is not prohibitive (electron current $70 \mathrm{~mA}$ ), makes possible ep collisions with a luminosity $10^{33} \mathrm{~cm}^{-2} \mathrm{~s}^{-1}$. How this is

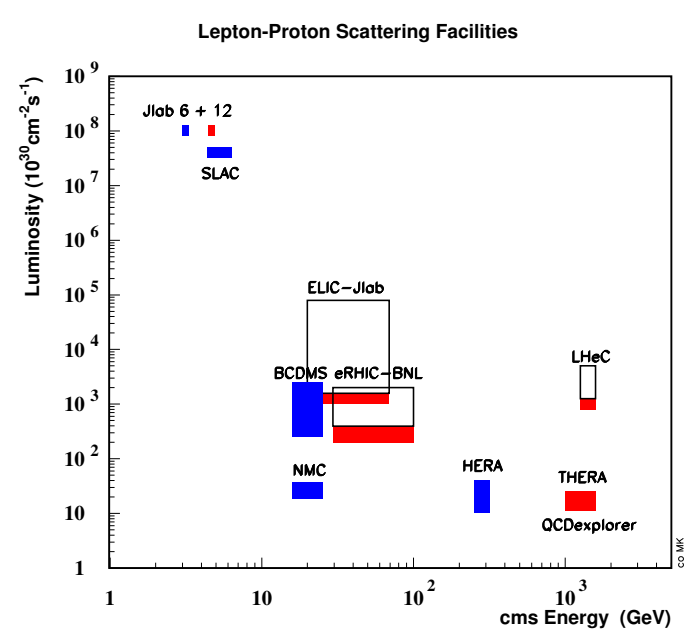

Figure 2: Luminosity achieved or foreseen at high energy, lepton-hadron, experiments; THERA and QCD-explorer are possibilities involving presently available hadron beams at HERA and the LHC which use "single-pass" linear collider electron beams; $\mathrm{LHeC}$ is described here; the estimated luminosity at $\mathrm{LHeC}$ is notable.

done is described in section 3 below, and in much more detail in [5]. Such a luminosity exceeds that achieved at all lepton-hadron experiments so far, except those at SLAC in 1969 (fig. 2). Though yet to be evaluated, it could also be the case that a purpose-built $70 \mathrm{GeV}$, $\mathrm{CW}, e$-linac in single-pass collision with LHC hadron beams could also achieve such luminosity at similar, or even lower, cost than an injection system and storage ring technology $[8]$.

It is thus far from fanciful to assume that $\mathrm{TeV} e p$ (and $e A$ ) physics at an $\mathrm{LH} e \mathrm{C}$ will be possible with both $\mathrm{TeV}$ interaction energy $(70 \otimes 7000 \mathrm{GeV})$ and adequate luminosity $\left(10^{33}\right.$ $\mathrm{cm}^{-2} \mathrm{~s}^{-1}$ ) for sub-attometric $e q$ physics.

The kinematic reach of a lepton-hadron collider is best expressed in terms of the Bjørken- $x$

\footnotetext{
${ }^{\mathrm{b}}$ Henceforth we take it for granted that an electron linac, or an electron storage ring in the LHC tunnel, will also, like HERA, operate well as a positron linac or storage ring.
} 
and $Q^{2}$ variables ${ }^{\mathrm{c}}$, that is the momentum as a fraction of the incident hadron momentum and the 4momentum transfer squared in the interaction between the lepton and the struck parton in the hadron. Fig. 3 shows (in modest logarithmic fashion) the resulting kinematic reach of the $\mathrm{LHeC}(70 \otimes 7000 \mathrm{GeV})$ in comparison with previous experiments. The $\mathrm{LHeC}$ probes the electron-quark $(e q)$ interaction at more than $1 \mathrm{TeV}$ with a spatial resolution of $1 / 10$ am (fig. 1). For the deepest ep interactions with $Q^{2} \sim 1 \mathrm{TeV}^{2}$ and with $10^{33} \mathrm{~cm}^{-2}$ $\mathrm{s}^{-1}, 30$ events will be obtained for in a plausible data-taking year of $3 \mathrm{fb}^{-1}$ at the $\mathrm{LHeC}$. Furthermore, for Bjørken$x$ down to $10^{-7}$, the $\mathrm{LH} e \mathrm{C}$ probes $e q$ physics with sub-femtometric resolution $\left(Q^{2} \geq 1 \mathrm{GeV}^{2}\right)$ in low- $x$ interactions where the quark is enveloped in the proton's (or ion's) immense chromodynamic field energy. For such extremely inelastic (high $y$ ) ep interactions, luminosity is only an issue if one has difficulty in the face of a huge data-rate at small angles (less than about $10^{\circ}$ ) to the incident proton beam.

Attometric and sub-attometric, eq physics in an $\mathrm{LHeC}$, with a purpose-built detector, is thus a powerful new probe of the Standard Model (SM), both its electroweak and its strong sectors. Experience at HERA has taught us that it is possible to make precision measurements of a swathe of $e q \mathrm{SM}$ processes. In contrast with collisions in a $p p$ collider, the final state is kinematically over-constrained at the $e$-hadron level, and the asymmetry in colliding beam energies in the laboratory throws the important, final state, hadrons from the $e$-hadron interaction into the detector, whereas in the old fixed-target, lepton-hadron, experiments they dribble out through the target mass. Furthermore, and also like TeV $e^{+} e^{-}$physics, precision experiments are feasible in $e p$ and $e A$ physics at an LHeC. In neutral current SM interactions, transverse energy flow, measured with modern hadronic calorimetry is kinematically balanced against the transverse energy of the scattered lepton, which itself is measured with the precision possible using electromagnetic calorimetry. LHeC physics is truly precision physics.

It is instructive to compare the TeV physics, which is possible at the LHC in terms of the nature of the initial parton-parton interactions, with the $\mathrm{TeV}$ physics which is possible at the $\mathrm{LHe} \mathrm{C}$ in terms of the initial $e$-parton interaction. For a lepton momentum of $70 \mathrm{GeV}$, $e p$ interactions at the $\mathrm{LH} e \mathrm{C}$ include final state phase space corresponding to inclusive $p p$ at LHC for "parton-out-of-proton" inelasticity $y \leq 0.01$, that is for "parton-out-of-proton" momentum fraction $x \leq 0.01$ (Fig. 4a). Our knowledge of the parton density functions of

${ }^{\mathrm{c}}$ The definitions and physical interpretation of the Lorentz covariant variables inelasticity $y$, Bjørken- $x$ and $Q^{2}$ are to be found liberally distributed throughout other contributions to these proceedings! 

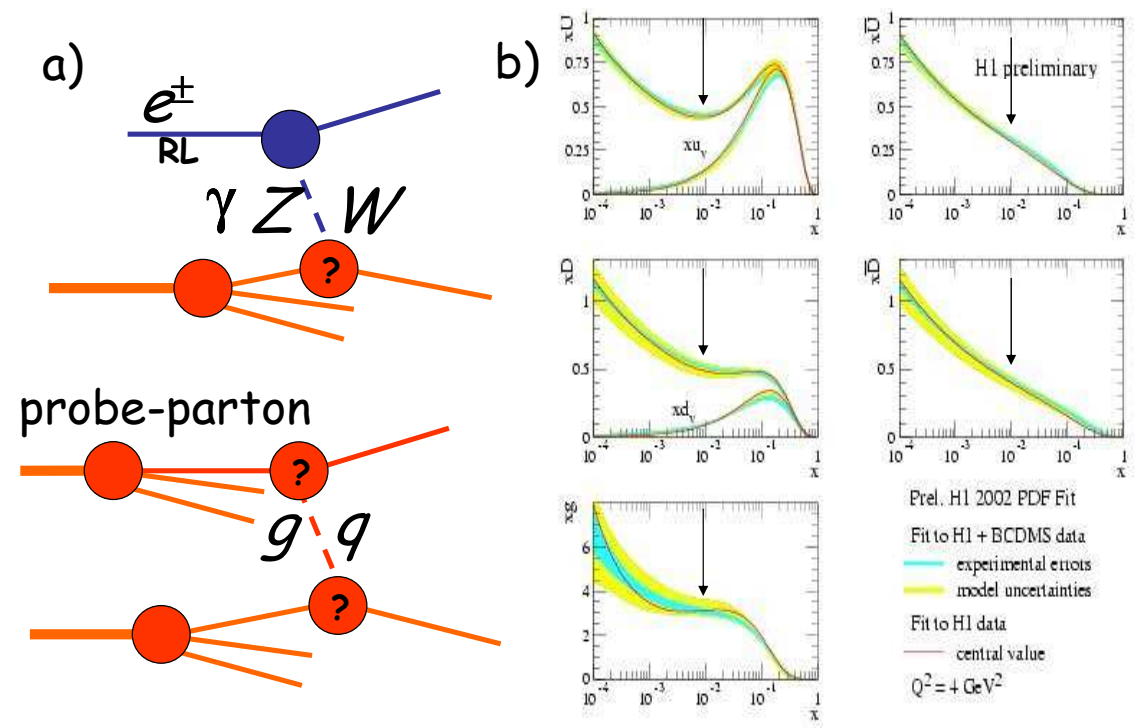

Prel. H1 2002 PDE Fit

Fit to Hl + BCDMS data

expetimental etrots

- rodel uncertainties

Fit to Hl data

- central value

$Q^{2}=+G e V^{2}$

Figure 4: a) Diagrams illustrating the lepton-parton level dynamics in $e p$ and in $p p$ interactions at respectively the $\mathrm{LHeC}$ (upper) and the $\mathrm{LHC}$ (lower); at the $\mathrm{LHeC}$ the incident $70 \mathrm{GeV}$ electron/positron $e^{ \pm}$(left $L$ or right $R$ handed, polarized, lepton) interacts electroweakly $(\gamma+Z, W)$ with a parton in the $7 \mathrm{TeV}$ beam; at the LHC the same phase space is covered by the inelastic interaction of a parton from one of the protons having fractional momentum Bjørken- $x=0.01$ with the other proton. b) Parton density functions (probability per unit $\ln x$ ) for $u$ and $d$ quarks and antiquarks, and for gluons, showing the mix of quark, antiquark and gluon at $x=0.01$ (marked with the arrows) at LHC; at $\mathrm{LHeC}$ this mix is replaced by the precision electroweak probe.

the proton, which specify the latter's structure in terms of the variety of possible partons, comes right now from measurements at HERA, and is summarized in Fig. 4b). For $x \leq 0.01$, $p p$ physics involves either a quark/antiquark or a gluon interacting with the other proton, roughly in a mix of gluon:(anti)quark of at least $\sim 2: 1$. Which of these, gluon or (anti)quark, is the probe is crucial to the interpretation of the physics, and discrimination will depend on final state jet topology and comparison with QCD and other $p p$-based simulation at the LHC. At $\mathrm{LHeC}$ the electroweak probe $(\gamma+Z$ for neutral current, $W$ for charged current) couples in an a priori well defined, and self-verifying, manner to each distinct flavour of quark, in which the consequences are immediately manifest in the neutral and charged current inclusive cross sections and their dependence on lepton helicity. It is primarily for this reason, taken with careful experimentation, that $\mathrm{LHe}$ C brings precision to $\mathrm{TeV}$ physics at the LHC. 

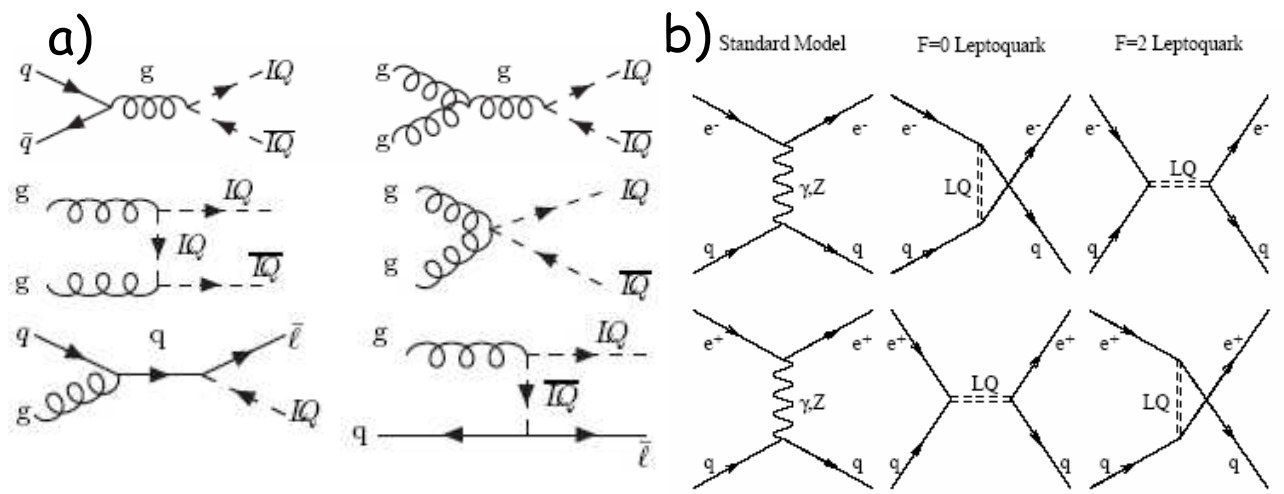

Figure 5: a) Leading order diagrammatic view of production mechanisms for new leptonquark (lepton $l$, quark $q$, gluon $g$, and leptoquark $t l Q$ ) physics in parton-parton interactions, and b) for SM and similar, new, electron/positron-quark physics.

The combination of this experimental precision and of the rather clean, "SM-and-beyond", $e q$ interaction mechanisms, means that experiments at $\mathrm{LH} e \mathrm{C}$ constitute a precise, $\mathrm{TeV}$-scale, discovery apparatus. Fig. 5 shows the simplest, leading order, parton-parton (a) and eq (b) processes in which new eq physics could be manifest. The new physics diagrams are displayed here in terms of "leptoquark" lines (LQ), and they can also be taken in a more generic sense given that new TeV-physics at the LHC, such as supersymmetry, could well be manifest as new lepton-quark dynamics at the $\mathrm{LHeC}$. If LQ lines are interpreted as a new leptoquark spectrosocopy, most models predict that at $1 \mathrm{TeV}$ the $e q \rightarrow \mathrm{LQ}$ formation cross section is 2 to 3 times larger than for similar, single, leptoquark production in parton-parton interactions.

By virtue of the electroweak nature of the deep-inelastic neutral current (NC) and charged current (CC) probes, sensitivity to signatures for new physics at $\mathrm{LHeC}$ is large because almost-completely-real, SM, amplitudes interfere with amplitudes for new physics (Fig. 5b). One can thus anticipate from precision analysis of the final state lepton $(e$ or $\nu)$ and jet(s) system, including correlation with initial state lepton helicity, a detailed quantification of new phenomena. For example, measurements of decay branching ratios and even spin-parity analysis of resonant $e q$ phenomena, both for fermion number $F=0\left(e^{+} q\right.$, i e meson) and for $F=2\left(e^{-} q\right.$ I e di-fermion). The potential for such measurements was amply demonstrated at HERA, where, with hindsight but for the misfortune of the energy scale of new physics being beyond the reach of $320 \mathrm{GeV}$ ep collisions, hitherto unchallengeable limits on a huge range of possibilities for new LQ physics have been obtained [9].

Of course, eq physics at the $\mathrm{LH} e \mathrm{C}$ is manifest in the environment of hadrons, be they protons or heavy ions. In a sense, this of course means that the $\mathrm{LH} e \mathrm{C}$ will be actually the world's most powerful and comprehensive microscope - attoscope - of matter! Many of the precision measurements which are possible will be of cross sections, in the form of for example hadronic structure functions, and new physics may well first show up as anomalies in such measurements.

To highlight the importance for discovery at the LHC of the $\mathrm{LHe}$ attoscope, one only has to look at fig. 6, which illustrates the magnitude of the task, in the face of SM background, 

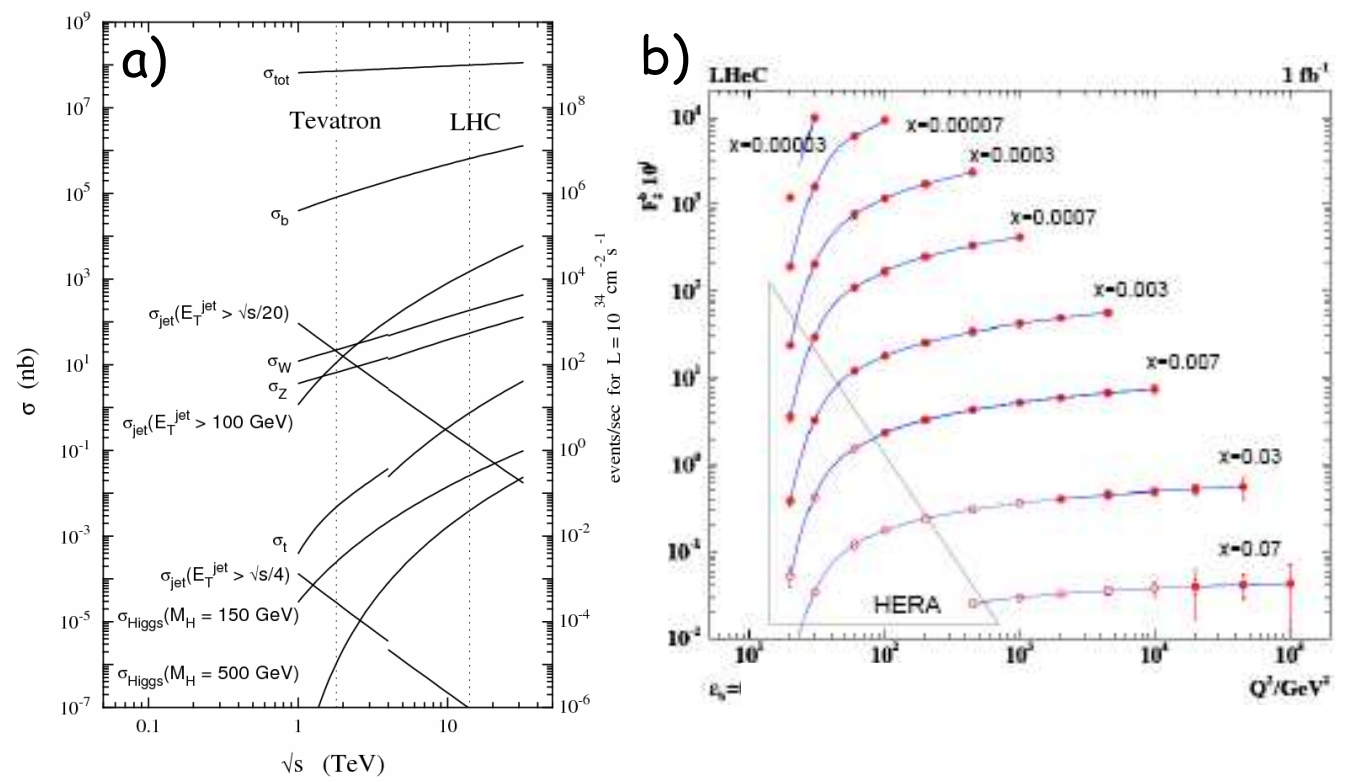

Figure 6: Total production cross sections at the LHC showing the challenge even for SM discovery; b) expectation for a measurement of $F_{2}^{b}$ at $\mathrm{LHeC}$, showing the huge increase in kinematic reach compared with HERA in Bjørken- $x$ and $Q^{2}$, and illustrating the impracticality of relying on extrapolation from HERA to LHC energy scales to obtain accurate enough $b$-quark pdfs for SM background subtraction if searching for Higgs production and $b$-quark decay.

of discovering and identifying the Higgs at the LHC by means of its $b$-quark decay mode. A comprehensive, and well quantified, understanding of the contribution of $b$-quarks to proton structure is a pre-requisite for a conclusive outcome. Extrapolation alone, however rigorously based, of knowledge from the HERA energy scale (see [10]) to that of LHC, is no substitute for direct measurement at the LHeC (and therefore LHC!) energy scale.

At low Bjørken- $x$ the relentless growth with decreasing $x$ of hadronic structure functions means that kinematics, coupled with experimental acceptance, govern the reach at $\mathrm{LHeC}$ for $e q$ physics (fig. 2). Luminosity only plays a role in probing rare exclusive processes at low- $x$. According to fig. 3 , it is possible at $\mathrm{LHeC}$ to probe, with a precision better than

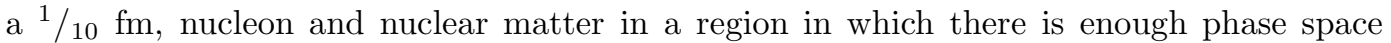
for on average up to about 5 partons to be resolved and disturbed in the chromodynamic field of the nucleon (compared with about 2 at HERA). This is an environment where the non-abelian, interaction of the chromodynamic field quanta, the gluons, plays a crucial role, and the $\mathrm{LHe}$ provides the only way to probe it with precision.

Gluon-gluon, gluon-quark, gluon-antiquark, and quark-antiquark dynamics will thus be essential pieces in the jigsaw which makes up the physics programme at the $\mathrm{LH} e \mathrm{C}$. In fact HERA opened our eyes to the realization that the deep-inelastic probe at low- $x$ constituted a completely new window on discovery and understanding of chromodynamic mechanism in both hadron structure and hadron interaction, and thereby on their inter-relationship. 
For the first time it was possible to measure simultaneously both structure and dynamics in terms of partons. Just as for proton structure pre-HERA, the outcome has been seminal, showing the way towards understanding the hadron-hadron interaction cross section at high energy in terms of chromodynamics. One begins to see that the grand horizon of building a universally applicable, chromodynamic, approach to the strong interaction in hadron and nuclear physics could be possible.

The $\mathrm{LHeC}$ takes us way beyond HERA in this physics. The magnitude of gluon density in the hadronic target which is accessible at the $\mathrm{LH} e \mathrm{C}$ may be such as to reveal new degrees of freedom in chromodynamic excitation, such as instantons. The LHeC is likely to put us in a regime where first gluon-gluon, and then other parton-parton, interactions are at a rate where they suppress parton densities at low- $x$ through gluon-gluon and quark-antiquark

a)

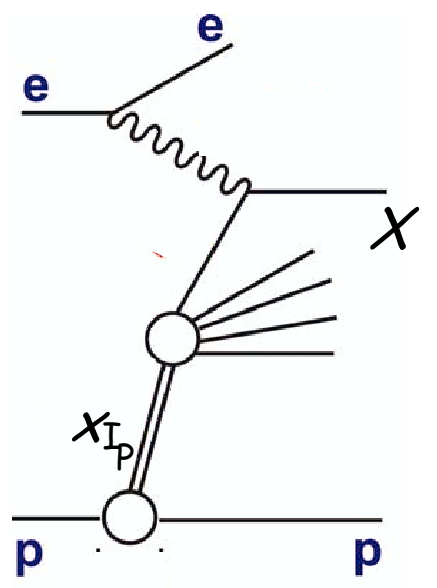

b)

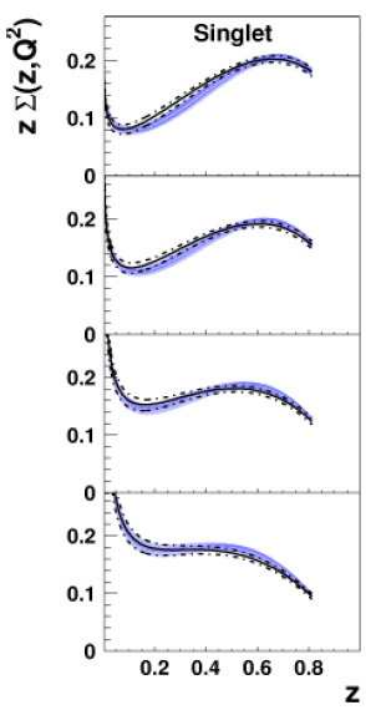

H1 2006 DPDF Fit A

(exp. error)

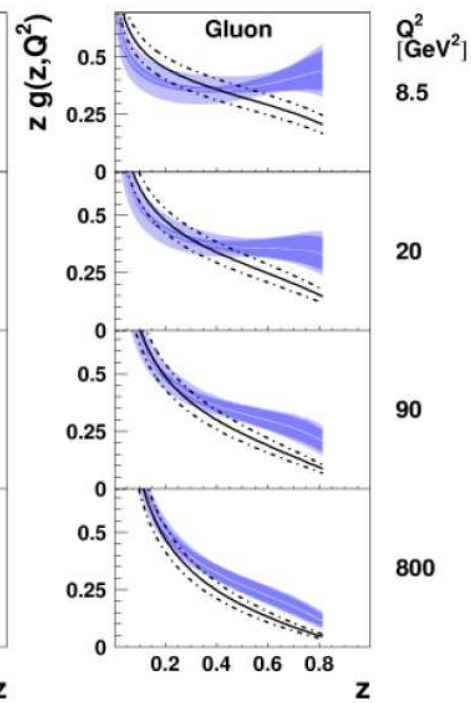

- H1 2006 DPDF Fit B ..... (exp.+theor. error)

Figure 7: a) Deep-inelastic diffraction $(e p \rightarrow e X p)$, in which, in low- $x$ deep-inelastic $e p$ scattering, the electroweak current couples to a quark and probes the diffractive interaction of a colour-singlet centre in the proton with small fractional momentum variable $x_{I P}$, that is in which, in the assumption of the dominance of pomeron exchange, the electroweak current probes the structure of the pomeron. b) Parton density functions for quarks (anti-quarks) and for gluons ( $z$ is the fractional momentum variable) extracted from the application of a "next-to-leading order", perturbative, QCD analysis of the structure function for deepinelastic diffraction; this amounts to the structure function of the pomeron if diffraction at the $\operatorname{chosen} x_{I P}$ is taken to be dominated by this Regge trajectory with leading vacuum quantum numbers. Note the different scale of ordinate for quark and for gluon, and also note the "leading gluon" nature of diffractive structure in which the quark density resembles closely that expected from gluon splitting and in which substantial gluon density persists to larger values of $z$. 
fusion, $g g \rightarrow g$ and $q q \rightarrow g$ ("absorption", "recombination", or "shadowing"). Therefore for the first time we may see the long awaited saturation of the relentless rise of structure functions at low- $x$, and we may understand it in terms of evolution between the "current" (small $Q^{2} \rightarrow \infty$ ) and "constituent" (large $Q^{2} \rightarrow 0$ ) pictures of hadronic structure. In terms of hadron dynamics, we will then reveal how chromodynamics causes the rise of total hadronic cross sections at high energy to level off, without which physics cannot be unitary.

It can be argued that parton recombination has already been demonstrated in the phenomena of parton hadronisation in jet physics and of colour-singlet clustering, the latter of which has been observed at HERA in the form of mesonic contributions to hadron structure at low Bjørken-x. Through the rigour of asymptotic Regge theory, these clusters can be associated with colour singlet spectroscopy and with the Regge-pole based phenomenology of "soft" hadronic interactions of the last few decades. Already HERA has revealed, through deep-inelastic measurements of diffraction (fig.7a), the short distance mechanisms at play in diffractive physics in terms of colour-singlet, gluon-gluon, dynamics $(e p \rightarrow e X p)$ - it has identified the pomeron in terms of asymptotically free, chromodynamic, degrees of freedom (Fig. 7b) [11]. For quark-antiquark, colour-singlet, dynamics, it has exposed the leading meson phenomena which persist to larger $x_{I P}(e p \rightarrow e X n)[12]$.

At the $\mathrm{LHeC}$ the reach to even lower Bjørken- $x$ will reveal exactly how gluon-gluon and quark-antiquark dynamics underpin these colour-singlet phenomena and developments of them, such as multi-Regge dynamics and reggeon calculus [13], which are presently the remaining pieces in our present "soft" hadronic phenomenology. Put more prosaically, this amounts to probing colour-singlet clustering within, or interacting with, colour-singlet clusters, while also resolving this chromodynamic physics in terms of partonic degrees of freedom. At last a complete picture of the interplay of quarks, gluons, and hadrons within baryonic matter, including nuclei, will be possible. The results will also have immediate application to the physics of confinement in QCD.

Many new and powerful insights into hadron structure which are possible in ep physics at high energy have only recently been realised at HERA. Most notable has been the reemergence of deep-exclusive physics, such as inelastic Compton scattering $e p \rightarrow e \gamma p$ and vector meson (VM) production $e p \rightarrow e \mathrm{VM} p$, in which the simultaneous handles of $Q^{2}$ and of the 4-momentum transfer squared $t$ probe the full tomography of proton and nuclear structure [14]. For such proton tomography, the importance of meeting the challenge of the operation of forward proton detection in "Roman pot"-like detectors, in which both $t$ and Feynman- $x$ are measured, is thus further emphasized. At the $\mathrm{LHeC}$ one can thus foresee establishing a full picture of hadronic structure and interaction at high energy in terms both of the nature and properties of constituents, and of how these constituents are distributed within their parent structure. Sub-femtoscopic tomography may well provide the greatest sensitivity than otherwise to new, short distance, chromodynamic physics.

A sense of the impact of low- $x$ physics at the $\mathrm{LHe}$ Can be gauged from the diffractive event rate as a function of inelastic diffractive mass $M_{X}$ in $e p \rightarrow e X p$, to be found in fig. 8 . A huge event rate (hundreds per $\mathrm{pb}^{-1}$ at $150 \mathrm{GeV}$ ) will be available. This opens the physics of $0^{+}$(vacuum quantum number) excitation in hadronic matter to the same scrutiny as $1^{-}$ excitation of the vacuum in LEP/ILC, $e^{+} e^{-}$, physics. We shall be able to measure heavy flavour dynamics in vacuum-quantum-number clusters in nucleons and nuclei. In short, the $\mathrm{LHeC}$ takes the physics of diffractively produced systems to the same energy scale as that of the hadronic final state at the putative ILC, and moreover extends it to chromodynamic environments beyond that of just the proton.

DIS 2007 
All of this $\mathrm{LHeC}$ hadronic physics, and more, is essential stuff if a full understanding of the strong interaction in terms of chromodynamics is to be achieved. Furthermore, we must have this confidence of understanding if we are to make discoveries at the TeV scale, whether at the LHC, the $\mathrm{LHeC}$ or elsewhere. When taken together with the ubiquity of the role of the strong sector of the SM in cosmic evolution, from the interaction and stability, or otherwise, of hadrons and of atomic nuclei and their role in nuclear synthesis, to the earliest phases of the Big Bang, such an understanding is seen to be crucial for future progress. We must continue to anticipate that the only way in which our confidence in QCD will continue to grow will be to pursue relentlessly its validity in as wide a range of phenomena as possible. HERA opened our

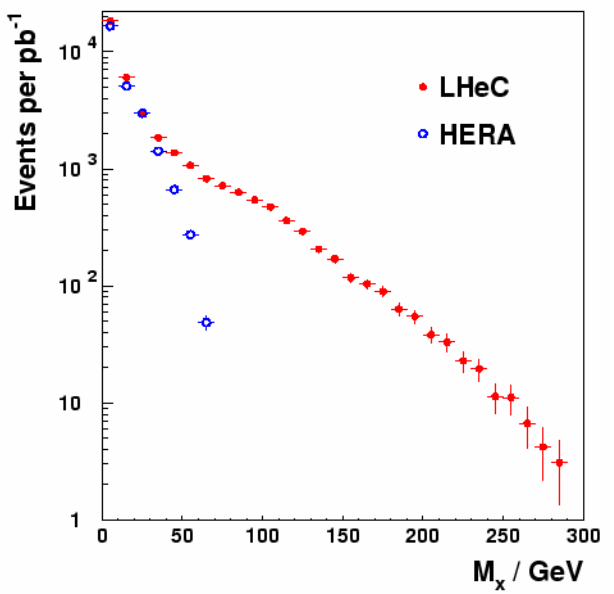

Figure 8: Event rate for deep-inelastic diffraction at the $\mathrm{LHe}$ C as a function of the diffractive dissociation mass $M_{X}$. eyes to the immense scope for testing chromodynamics in high energy $e p$ interactions, and it has provided seminal new insight into many long-standing and unresolved issues in strong interaction physics. The $\mathrm{LHeC}$ is likely to provide many more answers to many more questions, and some of these answers may amount to genuinely new chromodynamic discovery.

It must be clear from the few examples above that lepton-hadron interactions at the $\mathrm{LHeC}$ take us well into a new, TeV-energy, kinematic domain of eq physics, and that such access gives new, unique, opportunity for discovery and understanding. Put more starkly, the $\mathrm{LHeC}$ seems to be the only feasible way, both financially and technically, to make possible in a timely fashion the interaction of leptons with hadronic matter, namely quarks in various environments, at $1 \mathrm{TeV}$ or more energy. The $\mathrm{LHe}$ machine will thereby provide immense new insight into all sectors of the Standard Model through new discovery, and equally also through interpretation and distinction based on precision measurement.

\section{$3 \quad \mathrm{LH} e \mathrm{C}$ - the machine}

The $\mathrm{LHeC}$ is a pragmatic, and these days relatively modest, way to achieve a major step beyond the LHC in TeV-physics. By virtue of the unparalleled properties of the LHC hadron beams - proton and heavy ion, $7 \mathrm{TeV}$ energy, a new scale of intensity - a modest $70 \mathrm{GeV}$, $70 \mathrm{~mA}$, electron beam is required to achieve impressive luminosity. Here's how.

Given

- the LHC proton beam, namely bunches with $7 \mathrm{TeV}$ energy, each with $1.67 \times 10^{11}$ protons, of length $7.55 \mathrm{~cm}$,

- the inter-bunch spacing of $25 \mathrm{~ns}$,

- a normalized transverse emittance ${ }^{\mathrm{d}} \varepsilon_{p N}$ of $3.75 \mu \mathrm{m}$, and

\footnotetext{
${ }^{\mathrm{d}}$ An emittance $\varepsilon$ of a beam in a transverse dimension with Lorentz factor $\gamma$ is specified as $\gamma \varepsilon_{N}$ where $\varepsilon_{N}$
} 
- the experience of what really limits the current in an electron storage ring in the (once LEP) LHC tunnel, namely synchrotron radiation power loss defining the RF power consumption to be $50 \mathrm{MW}$, i.e. $28 \%$ of the CERN site load,

the well known formula for luminosity

$$
L=\frac{I_{e} N_{p} \gamma_{p}}{4 \pi e \varepsilon_{p N} \sqrt{\beta_{x p} \beta_{y p}}}
$$

gives $L=1.15 \times 10^{33}\left(\beta_{x p} \beta_{y p}\right)^{-1 / 2} \mathrm{~cm}^{-2} \mathrm{~s}^{-1}$. A more-than adequate ep luminosity thus looks possible (at of course a more-than-adequate CM energy!). For a plausible set of transverse $\beta$-functions, $\beta_{x p}$ and $\beta_{y p}$, of the proton beam, $L$ is more than a factor 10 larger than that of any lepton-proton experiment since that at SLAC in the late 1960s (fig. 1).

This result relies on the feasibility of colliding the electron or positron beam with the LHC proton (and ion) beams. Cutting short (undeservedly) work [5] of substantial technical detail to its barest conclusions, it turns out to be possible to establish LHC proton bunches in collision with bunches in a plausible electron ring in the LHC interaction region (IP) IP8. Table 1 summarises the main parameters which are necessary and which result, including a luminosity of $1.04 \times 10^{33} \mathrm{~cm}^{-2} \mathrm{~s}^{-1}$.

\begin{tabular}{|l|c|c|c|}
\hline Property & Unit & Leptons & Protons \\
\hline Beam Energies & $\mathrm{GeV}$ & 70 & 7000 \\
Total Beam Current & $\mathrm{mA}$ & 74 & 544 \\
Number of Particles / Bunch & $10^{10}$ & 1.04 & 17.0 \\
Horizontal Beam Emittance & $\mathrm{nm}$ & 25.9 & 0.501 \\
Vertical Beam Emittance & $\mathrm{nm}$ & 5 & 0.501 \\
Horizontal $\beta$-functions at IP & $\mathrm{cm}$ & 3.77 & 180 \\
Vertical $\beta$-functions at IP & $\mathrm{cm}$ & 4.44 & 50 \\
Energy loss per turn & $\mathrm{GeV}$ & 0.676 & $6 \cdot 10^{-6}$ \\
Radiated Energy & $\mathrm{MW}$ & 50 & 0.003 \\
\hline Bunch frequency / bunch spacing & $\mathrm{MHz} / \mathrm{ns}$ & $40 / 25$ \\
Center of Mass Energy & $\mathrm{GeV}$ & \multicolumn{2}{|c|}{1400} \\
Luminosity & $10^{33} \mathrm{~cm}^{-2} \mathrm{~s}^{-1}$ & \multicolumn{2}{|c|}{1.04} \\
\hline
\end{tabular}

Table 1: Basic LHeC machine parameters.

In IP8, the beams cross at $0.5 \mathrm{mrad}$ in the horizontal plane, for which the electronproton, beam-beam, interaction should not, and in this evaluation did not, in any way affect the $p p$ luminosity at IP1 (ATLAS), IP2 (ALICE), and IP5 (CMS) in LHC if one anticipates simultaneous $e p(e A)$ and $p p(A A)$ physics. The other hadron beam passes through the experiment displaced a few $\mathrm{cm}$ vertically above the interaction region beam pipe. At each of the other three IPs, two bypass tunnels for the electron beam are necessary $(2 \mathrm{~m}$ diameter with each section about $250 \mathrm{~m}$ long). Each tunnel links the main LHC tunnel to the IP survey shaft (fig. 9). In this way it seems to be possible at the LHC to have both $e p$ and $p p$ data-taking simultaneously (of course in different experiments!).

is the normalized emittance in that dimension. Here we have a situation in which emittance is taken to be the same in both transverse directions. 
The LHeC optics at IP8 leave many metres along the beam axes for experimentation, with access for detectors down to about $9.4^{\circ}$. For the low- $x$ physics programme (see above), where luminosity is not a limitation, low$\beta$ quadrupoles could be removed to facilitate "forward" and "backward" detectors at smaller angles, down to about $1^{\circ}$. The details of the optics for an interaction region for such low- $x$ data-taking remain to be resolved.

Longitudinally polarized (helicity) electrons and positrons in the $\mathrm{LHeC}$ increase substantially sensitivity to new eq physics both in the electroweak propagators and couplings and, more generically, in unforeseen discovery and its interpretation. One of HERA's remarkable achievements was to produce substantial, useful, polarisation using the Sokolov-Ternov effect [15] and "spin-rotation". In essence the synchrotron radiation in a storage ring generates a transverse polarisation for the stored electrons or positrons during the fill (Fig. 10a). As it develops, this polarisation can be rotated to be longitudinal before the $e p$ IP, and then back to transverse again after the IP [16]. The efficacy of the whole procedure depends largely on the alignment accuracy of the machine and the correct management of the coupling of spin motion to orbital motion such that dynamic equilibrium is achieved with minimal depolarizing effects. Already one can draw encouragement from the fact that transverse polarization could be produced in the LEP ring at $46 \mathrm{GeV}$, albeit with much patient optimization (fig. $10 \mathrm{~b}$ ). It will thus be important from the start to include the requirement of polarisation in the design of the electron ring lattice in the LHC tunnel. If, after all, a CW linac e-beam turns out to be a simpler and cheaper option than a storage ring, polarization may then be an easier issue for electrons and only slightly more of a challenge for positrons, but this has yet to be evaluated.

The first results from the evaluation which are presented above, and in more detail in [5], look extremely promising. Following completion of the details of the design and subsequent approval for construction, the realisation of an $\mathrm{LHeC}$ will most likely take a decade, in which the next "milestone" should be the completion of a Letter of Intent in the next two years (for 2009), and then, as soon as possible afterwards, a Technical Design Report ${ }^{\mathrm{e}}$.

By the time $\mathrm{LH} e \mathrm{C}$ data-taking is established, meeting the challenge at the LHC of further increases in hadron beam intensity will be underway. Thus the $\mathrm{LHe} \mathrm{C}$ has a unique long term potential both of luminosity growing beyond $10^{33} \mathrm{~cm}^{-2} \mathrm{~s}^{-1}$ and of an ep CM energy which will surely remain unsurpassed for a substantial fraction of this century.

eThis time-line bears an uncanny resemblance to the TeVatron-HERA era, when the first HERA physics appeared in 1992 about a decade after the turn-on of the TeVatron! 

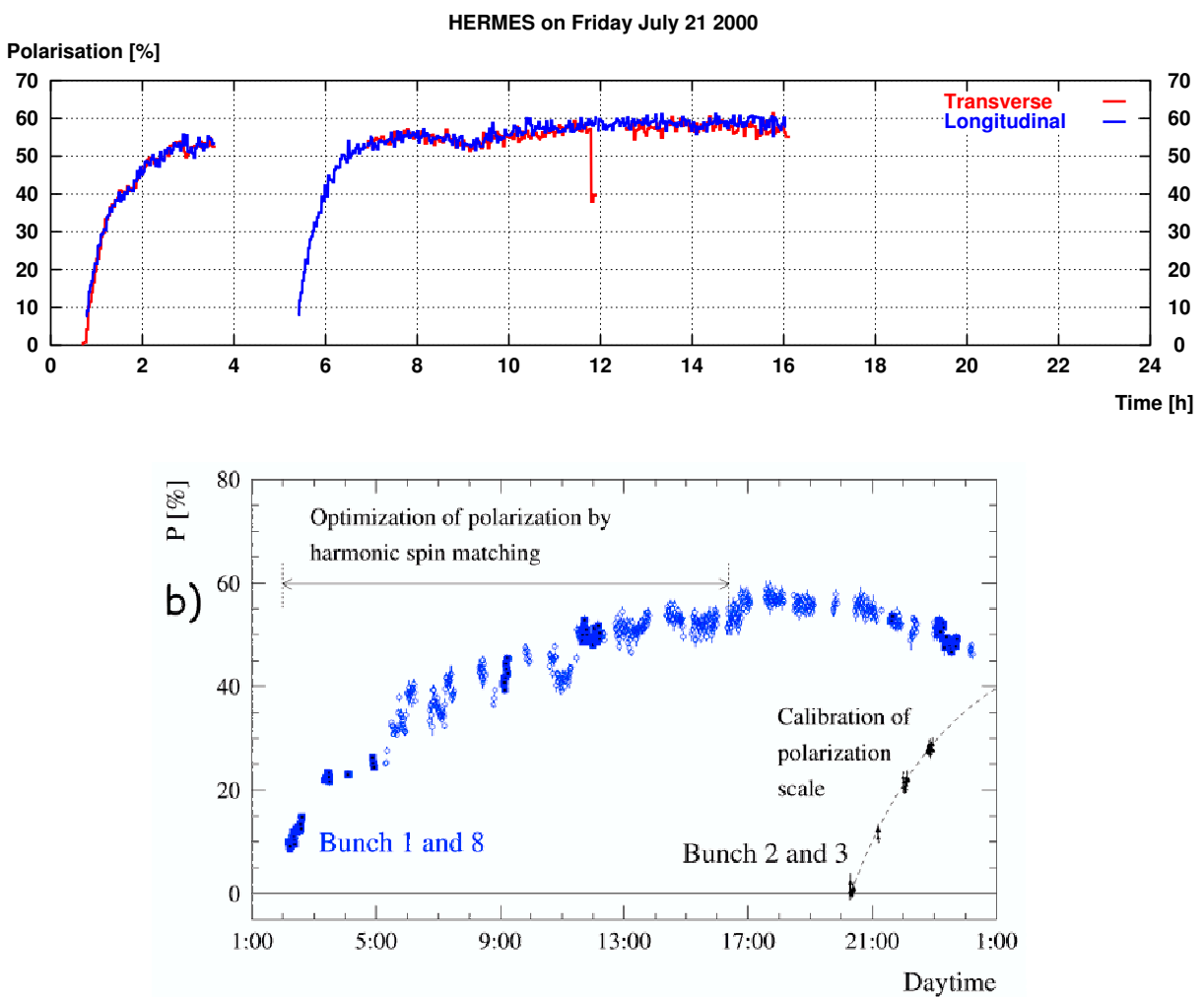

Figure 10: a) Development of transverse, and longitudinal polarization after "spin rotation", measured in the HERA ring at DESY in two positron fills; b) similar growth of transverse polarization measured in the LEP ring at CERN at $46 \mathrm{GeV}$.

\section{Experimentation at the $\mathrm{LH} e \mathrm{C}$}

The major feature of the $\mathrm{LHeC}(70 \mathrm{GeV} \otimes 7 \mathrm{TeV})$ kinematics is the electron-hadron beam momentum asymmetry, giving rise to the $e p$ system moving in the proton beam direction with a Lorentz $\gamma$ of about 5 , compared with about 3 at HERA. This has the desirable feature that high- $y$ (low- $x$ ) scattered electrons are boosted to larger angles, and the undesirable feature that high $Q^{2}$ physics is boosted to more forward angles.

Thus the challenge at the $\mathrm{LHe}$ is detection in the "forward" (hadron beam) direction so as to resolve the expected jet structures at the TeV energy scale. For the option described in section 3 aimed at the highest luminosity, "forward" means down to about $10^{\circ}$, and the challenge is to improve on instrumentation at HERA using the developments of the last 15 years in segmented track and calorimeter detectors.

For low- $x$ physics, the challenge is to achieve multi-particle reconstruction as close to the beam pipe as possible, while maintaining as precise as possible detection at larger angles and over the appropriate range of final state lepton and jet energies. The small angle (adjacent to the beam pipe) instrumentation must also be able to resolve "rapidity gaps" in hadron 
production if the wealth of colour-singlet, chromodynamic, physics is to be possible. For very forward production, one may be able to take advantage of the "Roman-pot"-like initiatives for CMS (TOTEM [18], FP420 [19]) and for ATLAS (FP420), which are now just beginning to face the reality of beam-pipe instrumentation in the full face of the LHC $p$-beam. Low- $x$ $e A$ physics poses similarly challenging, but often different, requirements for detection close to the beam-pipe.

It is not yet clear the degree to which an experiment optimized for $e p$ physics could also be optimized for $e A$ physics.

The timescale for completion of the $\mathrm{LH} e \mathrm{C}$ machine, which is outlined in section 3 above, is also essential if thorough design, $\mathrm{R} \& \mathrm{D}$, and optimization phases are to be carried out before construction of an experiment begins. Furthermore, it will be essential the specification for an experiment includes the requirement of handling the luminosity which can be foreseen throughout the lifetime of the LHC.

\section{Conclusion and Summary}

An $\mathrm{LHeC}$, consisting of a 70 to $100 \mathrm{GeV}$ electron or positron (e) beam in collision with the $7 \mathrm{TeV}$ proton and ion beams at the LHC, can be built. Its energy and its luminosity, when combined with the precision and acceptance which is possible with an experiment at an $e p$ collider, will bring both additional discovery and new understanding to future physics at the TeV energy scale.

The specifications for an electron and positron storage ring with which the necessary ep luminosity could be obtained at one LHC interaction point (IP) are realisable. They include the maintenance of $p p$ and $A A$ physics together with $e p$ physics by means of an appropriate bypass for the e-beam in each of the other LHC IPs. The LHeC luminosity will increase further with improvements in the LHC hadron beam intensity.

The $\mathrm{LHeC}$ is therefore an upgrade of the LHC facility at CERN, and is not a new, multibillion euro, global project. If constructed, it will stay at the "cutting-edge" for at least as long as does the LHC, taking data simultaneously with $p p$ and heavy ion experiments.

The physics programme at the $\mathrm{LHeC}$ will be pivotal in the era of $\mathrm{TeV}$ physics at the $\mathrm{LHC}$ and the discoveries there which will change the face of physics in the $21^{\text {st }}$ century, much as lepton-proton physics has been pivotal throughout the development of the Standard Model in the $20^{t h}$ century. There is no other way foreseen at the moment, other than with the $\mathrm{LHeC}$, to investigate the direct interaction of leptons and quarks at the TeV energy scale. The $\mathrm{LHe}$ is thus crucial to the pursuit of an understanding of one of the most important questions in physics, namely why are there both leptons and quarks?

\section{Acknowledgements}

I wish to thank my immediate colleagues, Des Barber, Max Klein, Paul Newman, Emmanuelle Perez, and Ferdy Willeke with whom this work has so far been completed. I hope that the opportunities, which are presented by the possibility of an LHeC, will encourage many to join in and to work with CERN with a view to the realization of an exciting new round of lepton-hadron physics at the TeV energy scale in parallel with hadron-hadron physics at the LHC.

I also wish to thank many other colleagues, too numerous to name, with whom for more than two decades I have been privileged to work at DESY. This year, 2007, HERA concludes 
its data-taking phase, and DESY takes its final steps after half a century towards becoming a "light source" laboratory. It is therefore appropriate here to express my gratitude for the scientific excellence of the laboratory which has underpinned the careers of many worldwide, including myself, in Particle Physics.

Many new, and not so new, colleagues at the Cockcroft Institute and at CERN have kept my enthusiasm for $\mathrm{LHeC}$ from clouding the challenges which such a machine poses for accelerator physics.

Without the substantial help of the DIS07 editorial team, my contribution to these proceedings would not have been possible, for which I am also very grateful.

\section{References}

[1] M. Gell-Mann, Phys. Lett. 8 (1964) 214

G. Zweig, "An $\mathrm{SU}_{3}$ Model for Strong Interaction Symmetry and Its Breaking", CERN Report TH-412 (Geneva 1964), and "Fractionally Charged Particles and $\mathrm{SU}_{6}$ ", in Symmetries in Elementary Particle Physics (Academic Press, New York, 1965) 192.

[2] J. D. Bjørken, Phys. Rev. 179 (1969) 1547, Phys.Rev. 163 (1967) 1767

R. P. Feynman, unpublished calculations for SLAC end station A experiment (1969), and Phys. Rev. Lett. 23 (1969) 1415

M. Riordan, account of application of Feyman;s calculations to SLAC data, "The Hunting of the Quark" (Simon and Schuster 1987) 148-155

[3] R. E. Taylor, Proc. $4^{\text {th }}$ International Symposium on Electron and Photon Interactions at High Energies (Liverpool, England, September 14-20, 1969), 251

E. D. Bloom et al., Phys. Rev. Lett. 23 (1969) 930

M. Breidenbach, PhD Thesis, MIT Laboratory for Nuclear Science Report MIT-COO-2098-635 (1970)

[4] A. Salam, "The Unconfined Quarks and Gluons", in Proceedings, XVIII International Conference on High Energy Physics, Tbilisi, USSR, July 1976

[5] J. B. Dainton, M. Klein, P. Newman, E. Perez, F. Willeke, "Deep Inelastic ElectronNucleon Scattering at the LHC", JINST 1 P10001, DESY-06-006, Cockcroft 06-05, http://xxx.lanl.gov/abs/hep-ex?papernum=0603016

[6] H. Abramowicz et al., TESLA TDR, DESY-2001-011, ECFA-2001-209 (2001); the THERA Book, DESY-LC-REV-2001-062 http://www.ifh.de/thera/

[7] D. Schulte and .F Zimmermann, "QCD Explorer", Proceedings of the Particle Accelerator Conference EPAC04, Lucerne, Switzerland, 2004

[8] S. Chattopadhyay, private communication

[9] H1 Collab., C. Adloff et al., Eur. Phys. J. C11 (1999) 447

[10] H1 Collab., A. Aktas et al., Eur. Phys. J. C45 (2006) 23

H1 Collab., A. Aktas et al., Eur. Phys. J. C40 (2005) 349

[11] H1 Collab., A. Aktas et al., Eur. Phys. J. C48 (2006) 715

H1 Collab., A. Aktas et al., Eur. Phys. J. C48 (2006) 749

[12] H1 Collab., C. Adloff et al., Eur. Phys. J. C6 (1999) 587

ZEUS Collaboration; S. Chekanov et al., Nucl. Physics B637 (2002) 3

ZEUS Collaboration; S. Chekanov et al. Nuclear Physics B776 (2007) 1

[13] V N Gribov, Zh. Eksp. Teor. Fiz. 53 (1967) 654

C. Edward Jones, F. E. Low, S.-H. H. Tye, G. Veneziano, J. E. Young, Phys. Rev. D6 (1973) 1033

[14] H1 Collab., C. Adloff et al., Phys.Lett. B517 (2001) 47

ZEUS Collaboration; S. Chekanov et al., Phys. Lett. B573 (2003) 46-62

H1 Collab., A. Aktas et al., Eur. Phys. J. C44 (2005) 1

[15] A. A. Sokolov, I F Ternov, Dokl. Akad. Nauk SSSR 153 (1963) 1052, [Sov. Phys. Dokl. 8 (1964) 1203]

[16] D. P. Barber et al., Phys.Lett. B343 (1995) 436

[17] R. Assmann et al., Proc. 11th Int. Symp. on High Energy Spin Physics, Bloomington, Indiana, USA, September 1994, Eds. K. J. Heller and S.L. Smith, AIP Conf. Proc. 343 (1995) 219

[18] V. Avati et al., EPJdirect A1 (2003) 1

DIS 2007 
[19] "FP420: an R\&D Proposal to investigate the feasibility of installing proton Tagging Detectors in the 420 m Region at the LHC" http://www.fp420.com/papers/fp420_UK_2006.pdf

[20] Slides:

http: //indico. cern. ch/contributionDisplay $\cdot$ py? contribId=271\&sessionId=10\&conf Id=9499 